\title{
Fusion Body Formation, Germ Tube Anastomosis, and Nuclear Migration During the Germination of Urediniospores of the Wheat Leaf Rust Fungus, Puccinia triticina
}

\author{
Xiben Wang and Brent McCallum
}

First and second authors: Cereal Research Centre, Agriculture and Agri-Food Canada, 195 Dafoe Rd., Winnipeg, MB, Canada R3T 2M9. Accepted for publication 3 July 2009.

\begin{abstract}
Wang, X., and McCallum, B. 2009. Fusion body formation, germ tube anastomosis, and nuclear migration during the germination of urediniospores of the wheat leaf rust fungus, Puccinia triticina. Phytopathology 99:1355-1364.

Vegetative or parasexual recombination is thought to be a key mechanism for the genetic diversity of cereal rust fungi. The process of germ tube fusion leading to hyphal anastomosis and nuclear recombination was analyzed in wheat leaf rust fungus, Puccinia triticina. Germ tube anastomosis was observed in 27 P. triticina isolates, each representing a different virulence phenotype. Germ tube fusion bodies (GFBs), which appeared as viscid globules formed at tips of germ tubes, were essential for germ tube anastomosis. The formation of GFBs was affected by the urediniospore density and the length of illumination during germination. GFBs were formed at the highest frequency when urediniospores were spread to a concentration of $1 \times 10^{6}$ urediniospores $/ \mathrm{ml}$ and incubated in dark for 12 to $24 \mathrm{~h}$ during germination. GFB attached to
\end{abstract}

ABSTRACT either the side of another germ tube ("tip to side") or to another GFB formed at the tip of a second germ tube ("tip to tip"). In "tip to side" anastomosis, two nuclei in the germ tube bearing the GFB migrated into the second germ tube through the GFB which resulted in four nuclei within this germ tube. In "tip to tip" anastomosis, nuclei in both germ tubes migrated into the fused GFB and all four nuclei came into close proximity. Urediniospores of isolates MBDS-3-115 and TBBJ-5-11 were stained with DAPI (4',6'diamine-2-phenylindole) and Nuclear Yellow (Hoechst S769121), respectively, and then mixed and germinated on water agar. Some fused GFBs contained nuclei stained with DAPI and nuclei stained with Nuclear Yellow in close proximity, demonstrating the fusion between genetically different $P$. triticina isolates. In some fused GFBs, "bridge-like" structures connecting different nuclei were observed.

Additional keywords: asexual recombination, chromosomal exchange, somatic recombination.
The wheat leaf rust fungus, Puccinia triticina Eriks, is one of the most economically important diseases on common wheat (Triticum aestivum L.) and durum wheat (Triticum turgidum L. var. durum) worldwide, causing up to $40 \%$ annual yield loss (12). The breeding of wheat cultivars with stable and long-lasting resistance to $P$. triticina has been very difficult to achieve due to the high variability present in $P$. triticina and its capability of migrating over large geographical regions (12). Forty to sixty unique $P$. triticina virulence phenotypes can be found annually in North America $(12,18,19)$. Therefore, it is important to understand the mechanism of virulence and genetic diversity in $P$. triticina. Such information is critical for the development of an effective strategy to manage this cereal disease.

Virulence variability in Puccinia species could originate from at least three possible sources including sexual recombination, mutation, and somatic hybridization (10). In North America, $P$. triticina reproduces on wheat by the production of asexual dikaryotic urediniospores. The susceptible alternate host, Thalicitrum speciosissimum, does not exist in Canada or the United States and native Thalicitrum species are generally resistant to $P$. triticina (29). Although some susceptible Thalicitrum hosts might be present, there is no evidence suggesting that the sexual phase of $P$. triticina is epidemiologically important or is a significant source of pathogenic variation in North America $(11,13)$.

Corresponding author: X. Wang; E-mail address: wangxib@agr.gc.ca

doi:10.1094/PHYTO-99-12-1355

( ) Her Majesty the Queen in Right of Canada, as represented by the Minister of Agriculture and Agri-Food Canada 2009
Mutation is believed to be the major source of variation in virulence of cereal rusts since virulence phenotypes arising from the same clonal lineage of Puccinia species often only differ in one or two virulence attribute(s) and are otherwise genetically similar (11,31). Although the precise mechanism involved is still not known, it has been hypothesized that new races of cereal rusts can develop through mutation followed by the selection for virulence against resistance genes deployed in wheat cultivars (11).

Empirical studies on $P$. graminis $(2,16,21,22)$, P. triticina (28), $P$. recondita (1), and $P$. striiformis $(9,15,33)$ have suggested the existence of somatic hybridization. Similarly, evidence for somatic hybridization has been obtained from virulence surveys. For example, $P$. graminis pathotype $34-2,11$ was believed to originate through somatic hybridization between two pathotypes (21-0 and $126-5,6,7,11)$ by a simple nuclear exchange event (31). The hybrid nature of $P$. graminis pathotype 34-2,11 was confirmed by comparing the isozymic and virulence profiles of $P$. graminis pathotype 34-2,11 and its potential parents (3).

It has been proposed that somatic hybridization in cereal rusts involves the anastomosis of hyphae followed by the reassociation of intact nuclei (heterokaryosis), or by the nuclear fusion and segregation (parasexuality and somatic meiosis) $(4,6,22)$. Hyphal anastomosis, a prerequisite for somatic hybridization and the parasexual cycle, has been reported in at least nine Puccinia species including $P$. triticina $(6,15,16,24,28,32)$. Rodenhiser and Hurd-Karrer (28) first demonstrated that round bodies designated as germ tube fusion bodies (GFBs) were formed at tips of some germ tubes when urediniospores of several Puccinia species, including $P$. graminis and $P$. triticina, were germinated on nutrientsolution agar. These GFBs were associated with germ tube 
anastomosis which resulted in a germ tube network during germination. A similar anastomosis process was also reported by Manners and Bampton (16) in P. graminis f. sp. tritici but without the formation of GFBs. However, the progress of germ tube anastomosis was only poorly described in those studies and whether the nuclear migration was involved in the germ tube anastomosis was not investigated. Subsequent studies done by Wilcoxson et al. (32) and Little and Manners (15) showed that two to four nuclei were commonly seen in GFBs. The existence of multinucleate conditions in GFBs was taken as evidence that germ tube anastomosis could be involved in the somatic hybridization of cereal rusts and contributed to the production of new cereal rust races. This hypothesis was supported by the findings of Nelson et al. (22) who reported that a new biotype which originated from somatic hybridization of two different $P$. graminis var. tritici races produced urediniospores or germ tubes with three or four nuclei. Nevertheless, the origin of these multinucleate GFBs and germ tubes has not been demonstrated and whether the nuclear exchange and reassortment are involved in the germ tube anastomosis in cereal rusts is still not known. Furthermore, it is not clear whether germ tube anastomosis in cereal rusts only occurs among genetically identical individuals or if it can involve genetically different isolates.

In summary, the actual mechanism of the somatic hybridization in cereal rusts and the significance of such a process in nature are not yet known due to the difficulty in detecting naturally occurring somatic hybrids and replicating the process under controlled conditions. The sequences of events leading to anastomosis and those that occur during the anastomosis are only poorly documented. Whether nuclear rearrangement and exchange occur during germ tube anastomosis and contribute to the production of new virulence phenotypes in $P$. triticina is a critical issue. The work reported here was done to investigate (i) the occurrence of germ tube anastomosis in $P$. triticina and conditions that affect germ tube anastomosis; (ii) the progression and stages of germ tube anastomosis from initial contact to hyphal fusion; and (iii) the nuclear migration and orientation in germ tube anastomosis.

\section{MATERIALS AND METHODS}

Characterizations of GFBs and anastomosis. Fresh urediniospores of $P$. triticina virulence phenotypes MBDS-3-115 and TBBJ-5-11 (19) were collected from pustules formed on leaves of wheat cultivar Thatcher. To achieve a repeatably low urediniospore density, the final concentration of urediniospore suspension was adjusted to $2.5 \times 10^{4}$ urediniospores $/ \mathrm{ml}$ with sterile distilled water in $1 \%$ Tween 20 (Sigma Canada, Mississauga). The well of a concave microscope slide (Fisher Scientific Canada, Mississauga) was filled with $100 \mu \mathrm{l}$ of $2 \%$ water agar containing $4 \%$ glucose. One microliter of urediniospore suspension $(2.5 \times$ $10^{4}$ spores $/ \mathrm{ml}$ ) was dropped onto the surface of $2 \%$ water agar containing $4 \%$ glucose and slides were dried in a laminar flow hood for $10 \mathrm{~min}$ to allow evaporation and facilitate the production of aerial germ tubes. Ten microliters of 1-nonanol solution (Sigma Canada) $\left(1.5 \mu \mathrm{l}\right.$ of 1 -nonanol/ml of acetone in $19 \mathrm{ml}$ of $\left.\mathrm{ddH}_{2} 0\right)$ was dropped at the edge of medium to remove the self-inhibitor of $P$. triticina urediniospore germination (7). The slide was then covered with a coverslip and the space between the microscope slide and the coverslip was sealed with $2 \%$ agar. Germ tube anastomosis was also observed in urediniospores germinated on agar plates, on microscope slides coated with $2 \%$ agar, and on wheat leaf surfaces. Germ tube anastomosis was followed over a 3- to 12-h period by observing germ tubes at regular intervals under a Leica DMRB microscope equipped with PL FLUOTAR $\times 10$ and $\times 20$ lens. To observe structures within GFBs, GFBs were fixed onto a transparent polymerase chain reaction (PCR) adhesive sealing film (Fisher Scientific Canada, Mississauga) and the film was cut to the size of $1 \times 1 \mathrm{~cm}^{2}$ and then mounted on microscope slides with $50 \%$ glycerol. To visualize GFBs and germ tubes formed on wheat leaves, $2 \mu \mathrm{l}$ of urediniospore mixture (MBDS-3-115 and TBBJ-5-11) at the concentration of $2.5 \times 10^{4}$ urediniospores/ml was dropped onto leaves of 3-week-old Thatcher plants. The inoculated wheat leaves were cut and incubated in petri plates. The moisture was maintained by placing a wetted paper tower in petri plates and petri plates were then sealed with Parafilm. The inoculated leaves were incubated in dark for $24 \mathrm{~h}$ and then cut into the size of $1 \times 1 \mathrm{~cm}^{2}$. Germ tubes and GFBs formed on leaf surfaces were stained with Trypan blue by dropping $100 \mu \mathrm{l}$ of $0.05 \%$ Trypan blue (Sigma Canada) onto the leaf surface. Stained leaf samples were mounted onto microscope slides with $50 \%$ glycerol. Structures within GFBs were observed under a Leica DMRB microscope equipped with PL FLUOTAR $\times 40$ and $\times 100$ oil phase contrast lens. Images were recorded with a Leica DFC290 digital camera and processed with Leica image application suite (2007) provided by the microscope manufacturer.

Effects of the urediniospore density and the length of the illumination period on the formation of GFBs. To investigate the effect of urediniospore density on the formation of GFBs, serially diluted urediniospore suspensions (isolates MBDS-3-115, TBBJ-5-11, and KBBJ-3-39) were spread onto $2 \%$ water agar plates containing $4 \%$ glucose ( $90 \mathrm{~mm}$ in diameter). One thousand, $400,100,10$, and $1 \mu \mathrm{l}$ of $P$. triticina urediniospore suspensions with concentrations of $1 \times 10^{3}, 2.5 \times 10^{3}, 1 \times 10^{4}, 1 \times 10^{5}$, and $1 \times$ $10^{6}$ urediniospores $/ \mathrm{ml}$ water, respectively, were spread onto agar plates using a glass rod. The total number of urediospore per plate was maintained at $1 \times 10^{6}$ urediospores per plate. Plates were dried for $30 \mathrm{~min}$ in the laminar flow hood to allow evaporation and facilitate the production of aerial germ tubes. Urediniospores were then germinated using the method described above. The number of GFBs, which appeared as viscid globules (10 to $15 \mu \mathrm{m}$ in diameter) forming at tips of germ tubes, was counted under microscope $24 \mathrm{~h}$ after the inoculation). The effect of the illumination period on the formation of GFBs was also investigated by placing agar plates spread with $2 \mu$ of urediniospore mixture $(1 \times$ $10^{5}$ urediniospores/ml) in dark for $0,3,6,9,12$, and $24 \mathrm{~h}$ and agar plates were placed under illumination provided by a 200 -watt Mazda soft white lamp. The number of GFBs was counted under microscope $24 \mathrm{~h}$ after inoculations.

Interisolate variation in the frequency of GFB formation. Twenty-seven $P$. triticina isolates, each representing a unique virulence phenotype, were collected previously during wheat leaf rust virulence surveys in Canada in 2003 and 2004 (18,19). These isolates were increased on 5-day-old wheat seedlings (cv. Little Club) pretreated with maleic hydrazide $(0.36$ g/liter, Sigma Canada, Mississauga) using the method described by McCallum and Seto-Goh (18). Freshly collected urediniospores were used throughout the study to ensure a high germination rate. A germination test was performed prior to every experiment and only urediniospore samples with higher than $95 \%$ germination rate were used.

Roughly $200 \mathrm{mg}$ of fresh urediniospores was suspended in $1 \mathrm{ml}$ of $1 \%$ Tween 20 solution in water in a $1.5-\mathrm{ml}$ centrifuge tube (Fisher Scientific Canada, Mississauga). The urediniospore suspension was then centrifuged and washed three times in $1.5 \mathrm{ml}$ of $\mathrm{ddH}_{2} \mathrm{O}$ with $1 \%$ Tween 20 (Sigma Canada). The final concentration of the urediniospore suspension was adjusted to $1 \times 10^{5}$ urediniospores $/ \mathrm{ml}$. Two microliters of urediniospore suspension was spread onto $2 \%$ water agar plates containing $4 \%$ glucose (90 $\mathrm{mm}$ in diameter, Fisher Scientific Canada). Inoculated agar plates were dried under a laminar flow hood for $30 \mathrm{~min}$ to allow evaporation. The moisture in agar plates was maintained by placing a filter paper wetted with $1.5 \mathrm{ml}$ of 1-nonanol solution inside the cover plate. The petri plate was then inverted and sealed with Parafilm. The agar plates with urediniospores were germinated in dark for $24 \mathrm{~h}$. The total number of germ tubes and GFBs 
(10 to $15 \mu \mathrm{m}$ in diameter) were counted to determine the proportion $(\%)$ of germ tubes with GFBs. For each isolate, five plates were prepared and each plate contained 10 drops of $2 \mu \mathrm{l}$ of urediniospore suspension. Averages from 50 replicates were used to construct the plot.

Nuclear migration in germ tube anastomosis. To track the nuclear migration during the germ tube anastomosis of a single isolate on the agar surface, urediniospores of $P$. triticina (MBDS$3-115$ ) (200 mg) were stained in $1.5 \mathrm{ng} / \mathrm{ml} \mathrm{4}, 6$-diamindino-2phenylindole (DAPI, Invitrogen Canada, Mississauga) by incubation for $2 \mathrm{~h}$ in a microcentrifuge tube (Fisher Scientific Canada) at room temperature. The urediniospore suspension was then centrifuged and pellets were washed six times with $1.5 \mathrm{ml}$ of $\mathrm{ddH}_{2} \mathrm{O}$ in $1 \%$ Tween 20 (Fisher Scientific Canada) and then resuspended in $200 \mu$ of $1 \%$ Tween 20 solution. The final concentration of the urediniospore suspension was adjusted to $2.5 \times 10^{4}$ urediniospores/ $\mathrm{ml}$ using $\mathrm{ddH}_{2} \mathrm{O}$ with $1 \%$ Tween and then germinated on $2 \%$ water agar plates containing $4 \%$ glucose, as described previously.

To observe the nuclear state in germ tubes and GFBs formed on leaf surfaces, urediniospores of $P$. triticina isolate MBDS-3-115 were stained with DAPI using the method described above. Then $2 \mu \mathrm{l}$ of DAPI-stained urediniospore mixture $\left(2.5 \times 10^{4}\right.$ urediniospores $/ \mathrm{ml}$ ) was dropped onto the leaf surface of 3-week-old Thatcher wheat plants. Leaves were then cut and incubated in dark in a sealed petri plate as describe above for $24 \mathrm{~h}$. Structures formed on the leaf surface were fixed onto a transparent PCR adhesive sealing film (Fisher Scientific Canada) and the film was then cut to $1 \times 1 \mathrm{~cm}^{2}$ and mounted on microscope slides with $50 \%$ glycerol.

For studying the nuclear migration during germ tube anastomoses involving two different isolates, DAPI-stained MBDS-3115 urediniospores were mixed with urediniospores of a second isolate, TBBJ-5-11, which were stained in $50 \mathrm{ng} / \mathrm{ml}$ Hoechst S769121 (Nuclear Yellow, Invitrogen Canada, Mississauga) for $2 \mathrm{~h}$ using a similar method. The final concentration of both urediniospore suspensions was adjusted to $2.5 \times 10^{4}$ urediniospores/ml. DAPI-stained MBDS-3-115 urediniospores were then mixed with Nuclear Yellow-stained TBBJ-5-11 urediniospores (180 $\mu$ l of MBDS-3-115 urediniospore mixture: $20 \mu \mathrm{l}$ of TBBJ-511 urediniospore mixture). The greater amount of DAPI-stained urediniospores in the mixture was used to increase the contrast between DAPI- and Nuclear Yellow-stained nuclei and facilitate the identification of hybrid fusion bodies with DAPI- and Nuclear Yellow-stained nuclei under microscope. The mixed urediniospore suspensions were then centrifuged and pellets were washed six times with $1.5 \mathrm{ml}$ of $1 \%$ Tween 20 solution and then resuspended in $200 \mu \mathrm{l}$ of $1 \%$ Tween 20 solution. Two microliters of the urediniospore mixture was germinated on $2 \%$ water agar containing $4 \%$ glucose and over 1,000 drops were made. Germ tube anastomosis was observed at regular intervals over 12-h periods using a Leica DMRB microscope equipped with a Leica Fibre Optic light source. For urediniospores stained with DAPI, fluorescence was observed with a single wave length Leica DAPI filter cube (excitation range $400 \mathrm{~nm}$ ) and images were recorded with a monochrome Leica DFC360FX camera. In the mixture of urediniospores stained with either DAPI or Nuclear Yellow, a Leica D filter cube (excitation filter BP355-425 and suppression filter LP 470) was initially used to examine the fluorescence emitted from DAPI and Nuclear Yellow. The DAPI-stained nuclei appeared light blue, whereas nuclei stained with Nuclear Yellow were bright yellow. The images were recorded with a color Leica DFC290 camera. When an anastomosis structure which contained both DAPI- and Nuclear Yellow-stained nuclei was identified, the fluorescence emitted from DAPI-stained nuclei was then recorded again with a monochrome Leica DFC360FX camera under a Leica DAPI filter cube which has a narrow excitation range for DAPI. The images were then overlaid using the Leica image application suite (2007) provided by the microscope manufac- turer. Images of germ tube anastomosis structures were also taken with a Leica monochrome DFC360FX camera using bright field microscopy.

\section{RESULTS}

Characterizations of GFBs and anastomosis. Germ tube anastomoses were observed when $P$. triticina urediniospores were germinated on the surface of either water agar (Fig. 1A) or wheat leaves (Fig. 1B). GFBs, appearing as round globules similar in size to urediniospores (10 to $15 \mu \mathrm{m}$ in diameter), were formed at tips of aerial germ tubes approximately $3 \mathrm{~h}$ after the germination of urediniospores on both the agar and leaf surfaces. Vigorous movement of brown granules inside germ tubes toward the ends of germ tubes was observed which resulted in the sudden formation of GFBs (less than $1 \mathrm{~min}$ ). This suggested the building up of internal pressure in germ tubes which resulted in the hyphal wall ballooning. Newly formed GFBs were very fragile and sometime broke on physical contact. However, their structure became hardened after a few hours.

More than $50 \%$ of urediniospores typically germinated $3 \mathrm{~h}$ after inoculation in vitro or on wheat leaf surfaces. Aerial germ tubes were formed on wheat leaf surfaces along with germ tubes that grew along surfaces on wheat leaves. Germ tubes and GFBs formed on the agar or wheat leaf surfaces have similar morphology. Since both germ tubes and GFBs were largely transparent, the identification of germ tube anastomosis on leaf surfaces was difficult without staining. Germ tubes tended to clump together after they were stained with Trypan blue solution which made counting GFBs difficult on leaf surfaces. Therefore, the frequency of germ tube anastomosis on wheat leaf surface was not determined. Nevertheless, similar morphology and the presence of multinucleate GFBs (picture not shown) on both the agar and wheat leaf surface suggests that the formation of GFBs is similar in vitro and on the surface of leaves.

Germ tubes bearing GFBs often bent downward, presumably because of weight of GFBs and GFBs sometimes burst or flatten out when they touch the surface of agar or wheat leaves. In some cases, germ tubes with GFBs reverted to the normal growth when they failed in contact with any other germ tubes. If a GFB came in contact with another germ tube, it attached either to the side of this germ tube or directly to another GFB (Fig. 1A). The growth of germ tubes bearing GFBs was arrested once contact with another germ tube or GFB occurred and the initial germ tube started to coil inside the GFB (Fig. 1C). The exchange of cytoplasm between two germ tubes inside the GFB was observed during the germ tube anastomosis (Fig. 1D). The migration of nuclei in germ tubes during the anastomosis was tracked through DAPI staining, a fluorescent dye specific to nuclei acid. Nuclei migrated from urediniospores into germ tubes upon germination. When germ tube anastomosis was absent, nuclei were randomly distributed in $P$. triticina germ tubes. Once a GFB contacted the side of another germ tube or a second GFB, nuclei migrated into the GFB. Two dikaryotic nuclei $(\mathrm{N}+\mathrm{N})$ were always present in the GFB attaching to either the side of another germ tube or to a second GFB.

Effects of the urediniospore density and length of illumination on the formation of GFBs. Both the spore density and length of illumination affected the formation of GFBs. GFBs were formed at the highest frequency when the urediniospore suspension was spread at a concentration of $1 \times 10^{6}$ urediniospores $/ \mathrm{ml}$, whereas only sporadic GFBs were produced from urediniospores spread at the concentration of $1 \times 10^{3}$ and $2 \times 10^{3}$ urediniospores/ml (Fig. 2A). When urediniospore suspension was spread at the concentration of $1 \times 10^{5}$ urediniospores $/ \mathrm{ml}$, a minimum of $6 \mathrm{~h}$ incubation in dark was sufficient for the production of GFBs and the highest number of GFBs was formed by urediniospores incubated in dark for 12 to $24 \mathrm{~h}$ during germination (Fig. 
2B). In addition, the formation of GFBs and subsequent germ tube anastomoses were completely suppressed by flooding agar plates with distilled water (Fig. 3B) or by constant illumination for $24 \mathrm{~h}$ during germination (Fig. 3C).

Interisolate variation in the frequency of GFB formation. The occurrence of germ tube anastomosis was investigated in 27 $P$. triticina isolates, each representing a unique virulence phenotype (Fig. 4). In all isolates, the formation of GFBs was observed during the germination of urediniospores. The percentage of germ tubes bearing GFBs varied from 1 to $14 \%$ and roughly 5 to $21 \%$ of the GFBs formed were involved in anastomosis. No correlation was found between the proportion of germ tubes bearing GFBs and specific virulence phenotype. Variations in the frequency of GFB formation were also observed between isolates with the same virulence phenotype (isolates MBDS-3-115 and TBBJ-5-11).

Nuclear migration in germ tube anastomosis. Germ tube anastomosis always involved at least one GFB. We were unable to find anastomoses between germ tubes without the involvement of GFBs. The GFB could fuse either to the side wall of a nearby germ tube ("tip to side") (Fig. 5A) or to another GFB when they came in contact ("tip to tip") (Fig. 5B). Nuclear migration during germ tube anastomosis was tracked through DAPI nuclear staining. In "tip to side" anastomosis, two nuclei from the germ tube bearing GFB migrated into the GFB after the contact between two germ tubes had been made (Fig. 6A and B). Then these two nuclei migrated from the GFB into the second germ tube (Fig. 6C to F) and resulted in a germ tube with four nuclei (Fig. 6G and H). In "tip to tip" germ tube anastomosis, nuclei from both germ tubes $(\mathrm{N}+\mathrm{N}$ plus $\mathrm{N}+\mathrm{N})$ were present in fused GFBs (Fig. 7A and B). Nuclei from two fusing germ tubes came into closer contact overtime in fused GFBs. Rearrangement of nuclei also appeared to occur when germ tubes inside GFBs started to fuse (Fig. 7C and D). A pairing configuration in which two of the four nuclei stayed closely paired was commonly seen in GFBs (Fig. 7E and F). Further examination of germ tubes participating in the anastomosis made it unlikely that four nuclei $(\mathrm{N}+\mathrm{N}+\mathrm{N}+\mathrm{N})$ within fused germ tubes or fused GFBs originated from mitosis since germ tubes which had not undergone anastomoses only had a dikaryotic nuclear configuration. A uniformed dikaryotic nuclear configuration $(\mathrm{N}+\mathrm{N})$ was also found in urediniospores and germ tubes germinated on agar plates flooded with water or under constant illumination where the formation of GFBs was suppressed and germ tube anastomosis did not occur. Furthermore, 'bridge-like' connections between two nuclei were observed in GFBs with four DAPI-stained nuclei, which originated from the urediospore mixture of MBDS-3-115, indicating that these nuclei could be associated during the anastomosis (Fig. 7G and $\mathrm{H}$ ). However, we were unable to differentiate the origin of these nuclei as to which parent germ tube they were coming from.

To investigate whether germ tubes from different $P$. triticina isolates can anastomoses, urediniospores of one isolate, MBDS-3115 , were stained with DAPI and urediniospores of a second isolate, TBBJ-5-11, were stained with Nuclear Yellow. Urediniospores of two isolates were then mixed and germinated on agar plates. Nuclei from urediniospores stained with DAPI displayed a light blue color, whereas nuclei from urediniospores stained with Nuclear Yellow appeared as bright yellow dots under fluorescence microscope (Fig. 8B and D). Germ tube anastomoses involving nuclei from each of two isolates were found in both "tip to side" (Fig. 8A and B) and "tip to tip" arrangements (Fig. 8C and D). In the "tip to tip" germ tube anastomosis, one DAPI-stained nucleus (isolate MBDS-3-115) and one Nuclear Yellow-stained nucleus (TBBJ-5-11) were present in close proximity and on the same plane. This suggested that nuclei from two genetically different $P$. triticina isolates could be paired during germ tube anastomosis.

\section{DISCUSSION}

Two patterns of germ tube anastomosis have been previously reported in cereal rusts, the anastomosis involving the formation

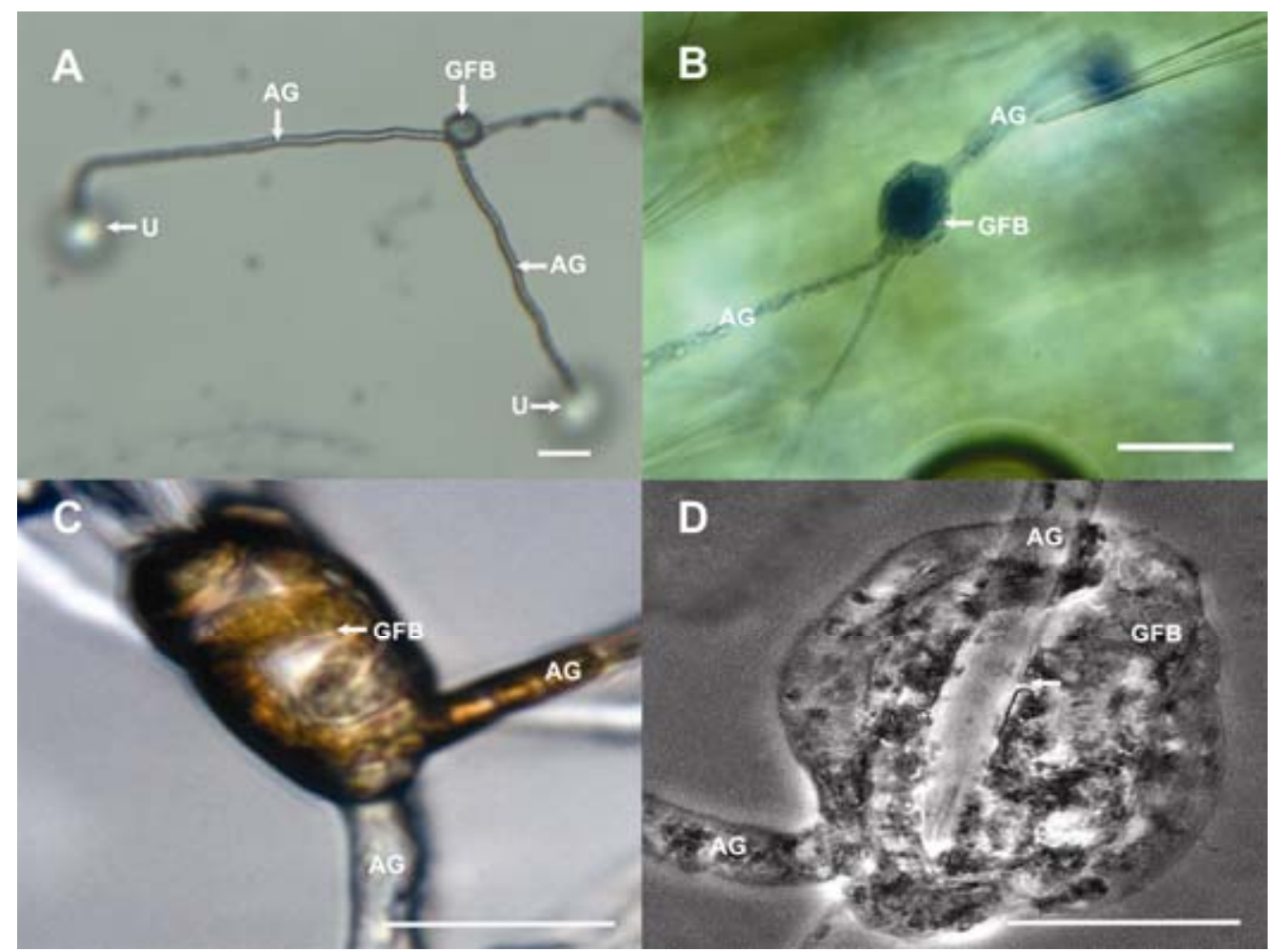

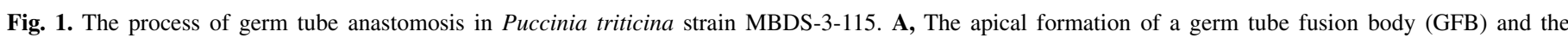

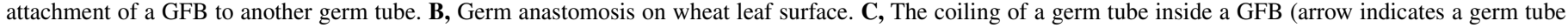

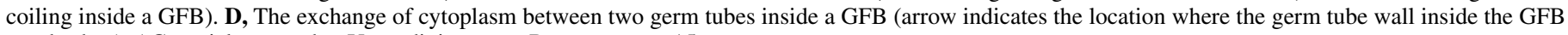
was broken). AG, aerial germ tube; $\mathrm{U}$, urediniospores. Bar represents $15 \mu \mathrm{m}$. 
of specialized GFBs (28) and anastomosis between two germ tubes directly without the formation of GFBs (16). In our study, germ tube anastomoses in P. triticina involved at least one GFB which was in agreement with the observation made by Rodenhiser and Hurd-Karrer (28). It is not clear why the anastomosis between two germ tubes without the involvement of GFB, as previously described by Manners and Bampton (16) in $P$. graminis, was not observed in our study. It was suggested by Manners and Bampton (16) that the oxygen content during the germination of urediniospores might affect the germ tube anastomosis in cereal rusts. Nevertheless, at least one GFB was involved in anastomosis when urediniospores of $P$. triticina were germinated either on the surface of agar or in concave microscope slides, conditions similar to those used by Manners and Bampton (16). This discrepancy could indicate that there are some differences between germ tube anastomosis in $P$. triticina and $P$. graminis.

The migration of nuclei during germ tube anastomosis was tracked through DAPI staining and fluorescence microscopy. Dikaryotic nuclei $(\mathrm{N}+\mathrm{N})$ migrated into germ tubes upon the germination of urediniospores and they were randomly distributed

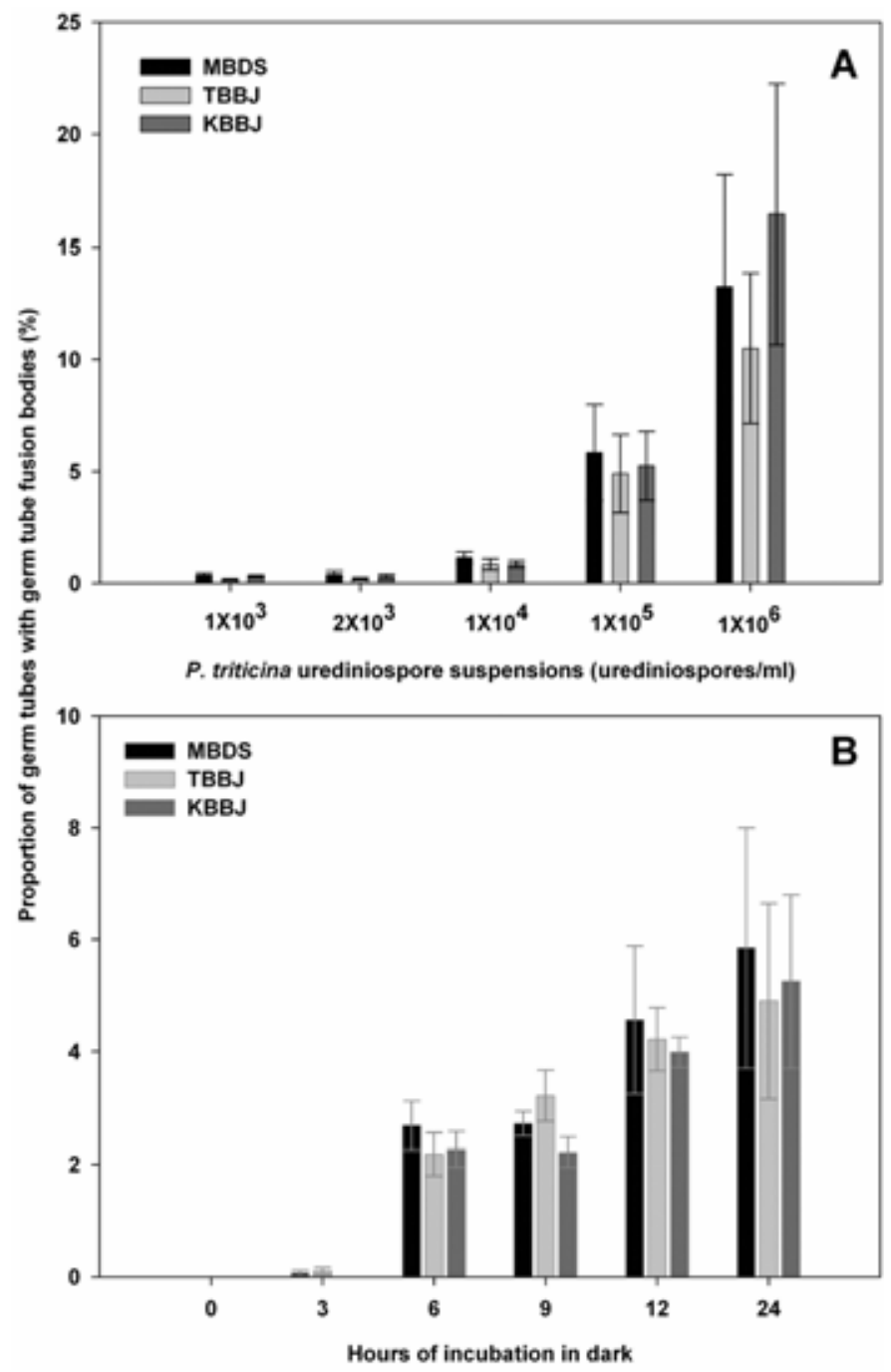

Fig. 2. The effect of the $\mathbf{A}$, urediniospore density and $\mathbf{B}$, length of illumination period on the formation of germ tube fusion bodies (GFBs) during the germination of Puccinia triticina urediniospores. Isolates with virulence phenotype MBDS, TBBJ, and KBBJ were used to determine the effect of urediniospore density on the formation of GFBs (the average determined from 10 replicates was used to construct the plot and error bars represent the standard error). The urediniospores mixtures were spread at a concentration of $1 \times 10^{5}$ urediniospores $/ \mathrm{ml}$ for the determination of the effect of the length of illumination period on the formation of GFBs. in germ tubes in the absence of GFB and germ tube anastomosis. However, both dikaryotic nuclei migrated into GFBs and were positioned in close proximity inside GFBs. Since it is known that fungal nuclei tend to migrate into germ tubes to achieve the relatively equal spacing upon the formation of germ tubes (8), the presence of two dikaryotic nuclei in the germ tube tightly packed inside the GFB suggests that the coiling of germ tube is related to

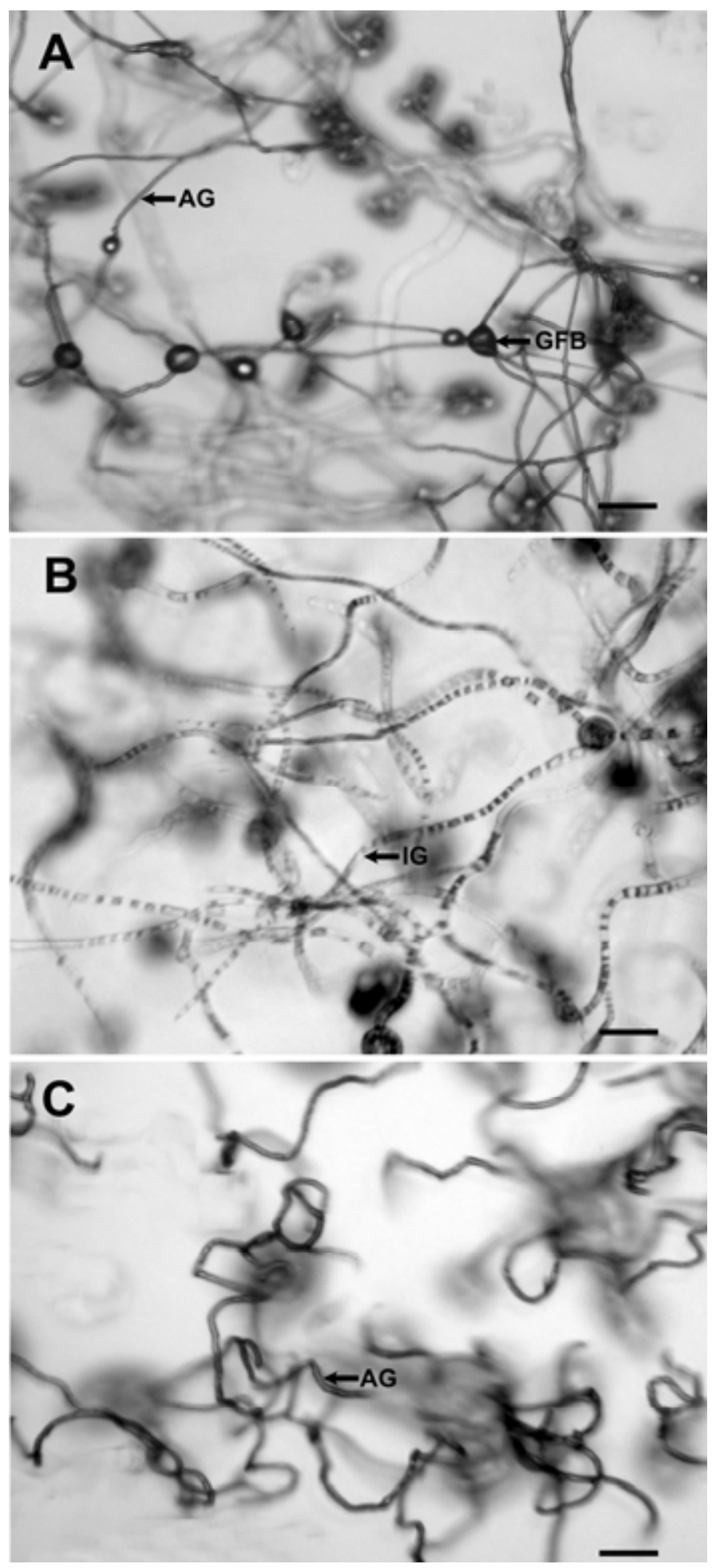

Fig. 3. The effect of flooding or constant illumination on the formation of germ tube fusion body (GFB) in Puccinia triticina strain MBDS-3-115. A, Urediniospores germinated in dark. B, Urediniospores germinated on agar plates in dark and flooded with water. C, Urediniospores germinated under constant illumination. AG, aerial germ tube; IG, immersed germ tube. Bar represents $50 \mu \mathrm{m}$. 
the migration of nuclei into close proximity with the second germ tube during the anastomosis.

Rodenhiser and Hurd-Karrer (28) reported that the formation of GFBs in Puccinia species was inhibited by illumination, and that short wave lengths at the blue end of spectrum were responsible for this inhibitory effect. Similar observations were also made in our study in which GFBs were not produced when $P$. triticina urediniospores were germinated under the constant illumination. However, a short period of incubation in dark ( 3 to $6 \mathrm{~h}$ ) during germination was sufficient for the induction of GFBs. This result indicates that illumination does not inhibit the formation of GFBs directly and instead it might affect the production of inducer for GFB formation. The existence of an extracellular inducer for the formation of GFBs is also supported by the observation that the formation of GFBs was completely suppressed by flooding urediniospores with water during germination in dark and the frequency of GFB formation was affected by the urediniospore density. The involvement of an extracellular inducer in the formation of conidial anastomosis tube (CAT), a specialized cellular element responsible for the anastomosis between conidia of Neurospora crassa and Colletotrichum lindemuthianum, has been recently proposed (27). Since the induction of CAT in $N$. crassa is dependent on the conidia density, a form of quorum sensing has been proposed for the regulation of CAT in which conidia monitor their population density by releasing signal molecules into environment $(26,27)$.

To date, anastomoses between different types of fungal spores and germ tubes have been described in 21 genera and 73 species (26) and this process is best characterized in the system of the conidial anastomosis in Colletotrichum and Neurospora which all

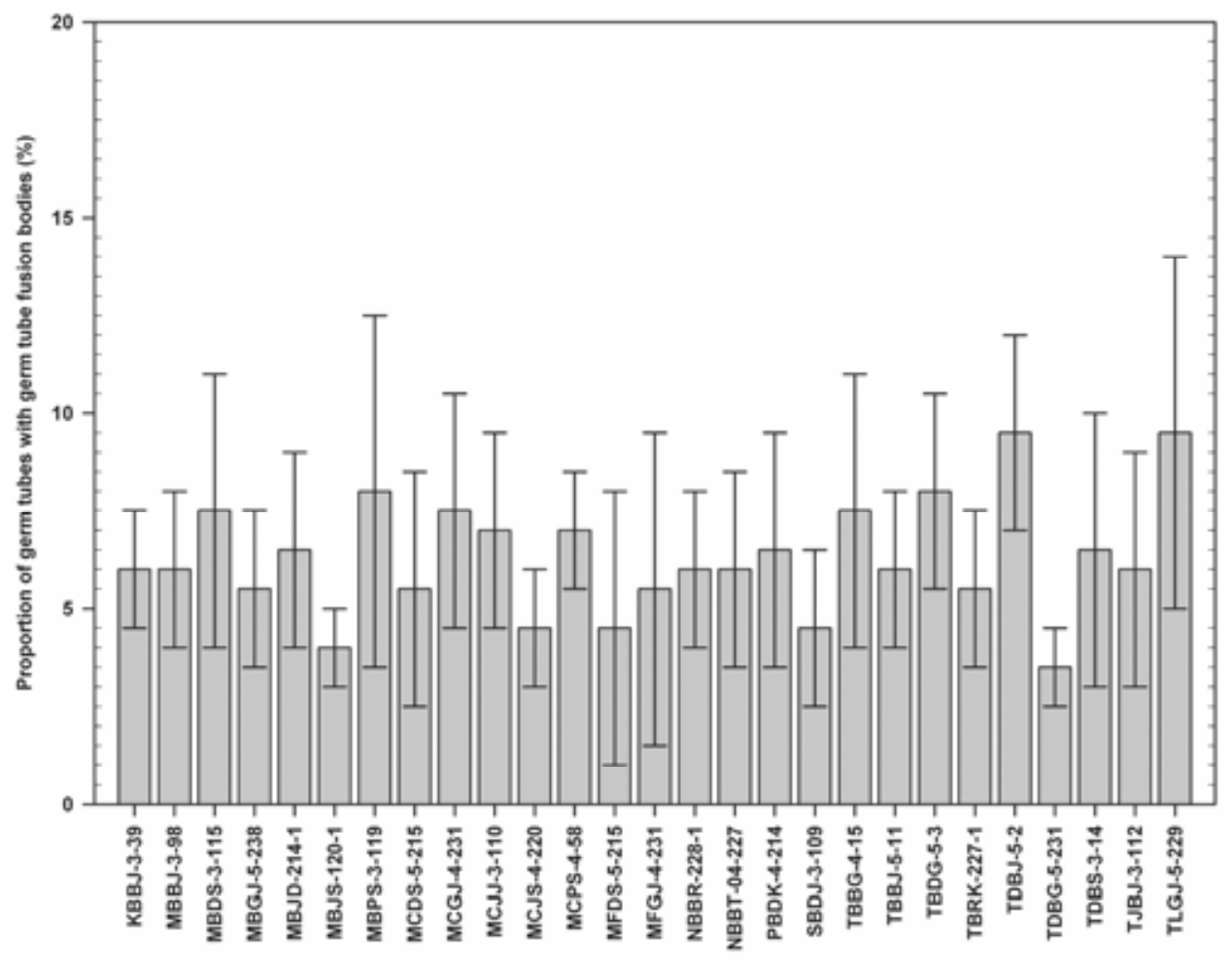

Virulence phenotype

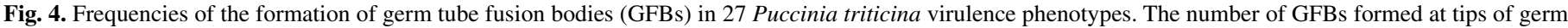
tubes was counted $24 \mathrm{~h}$ after germination (an average of 50 replicates was used to construct the plot and error bars represent the standard error).
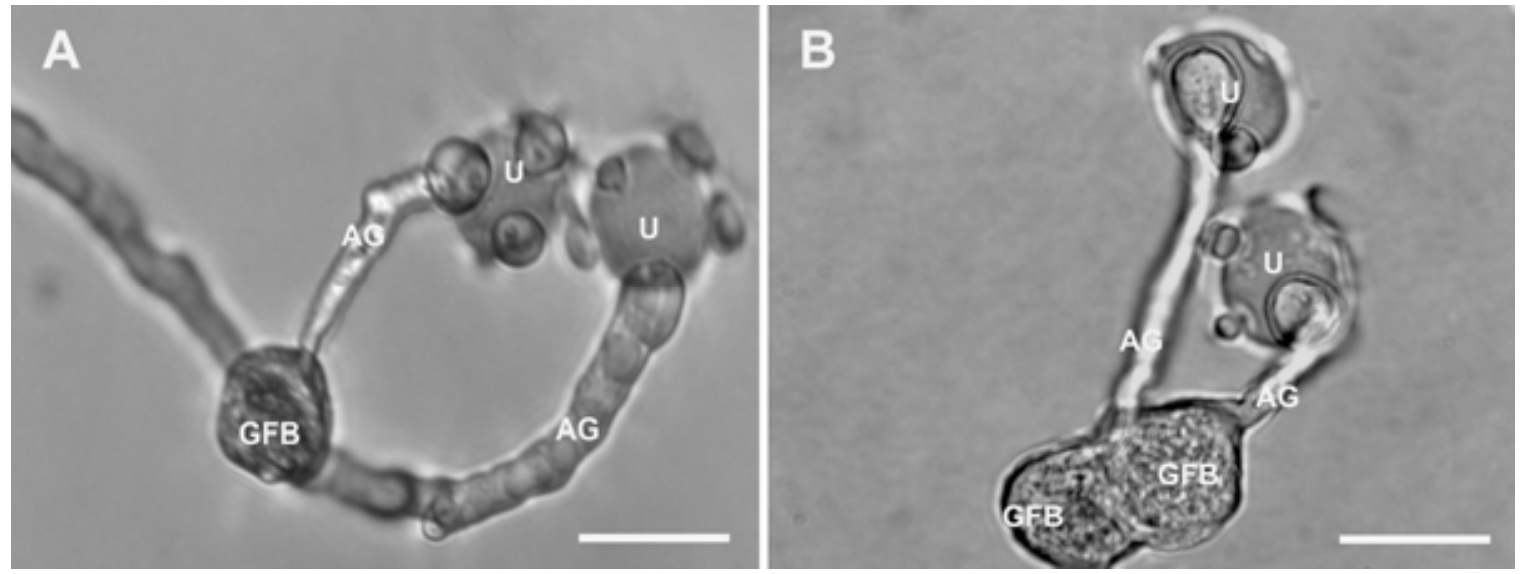

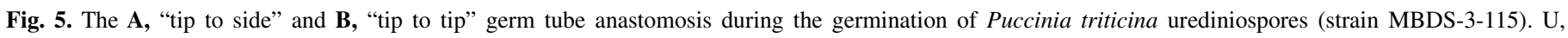
urediniospore; GFB, germ tube fusion body; AG, aerial germ tube. Bar represents $15 \mu \mathrm{m}$. 
involve specialized CATs (25-27). Although GFBs and CATs are both produced by germ tubes generated from asexual spores and involved in the anastomosis, there are several differences between GFBs and CATs. $P$. triticina GFBs are only produced at tips of $P$. triticina germ tubes, whereas CATs can originate from either conidial germ tubes or conidia directly. In addition, $P$. triticina
GFBs are capable of forming anastomosis with the side wall of another germ tube ("tip to side") or with another GFB formed at the tip of a second germ tube ("tip to tip") whereas CATs are much shorter, grow towards each another and only fuse tip to tip.

It has been repeatedly shown that new races of $P$. graminis (2,21), P. triticina (1), and $P$. striiformis (15) could arise by
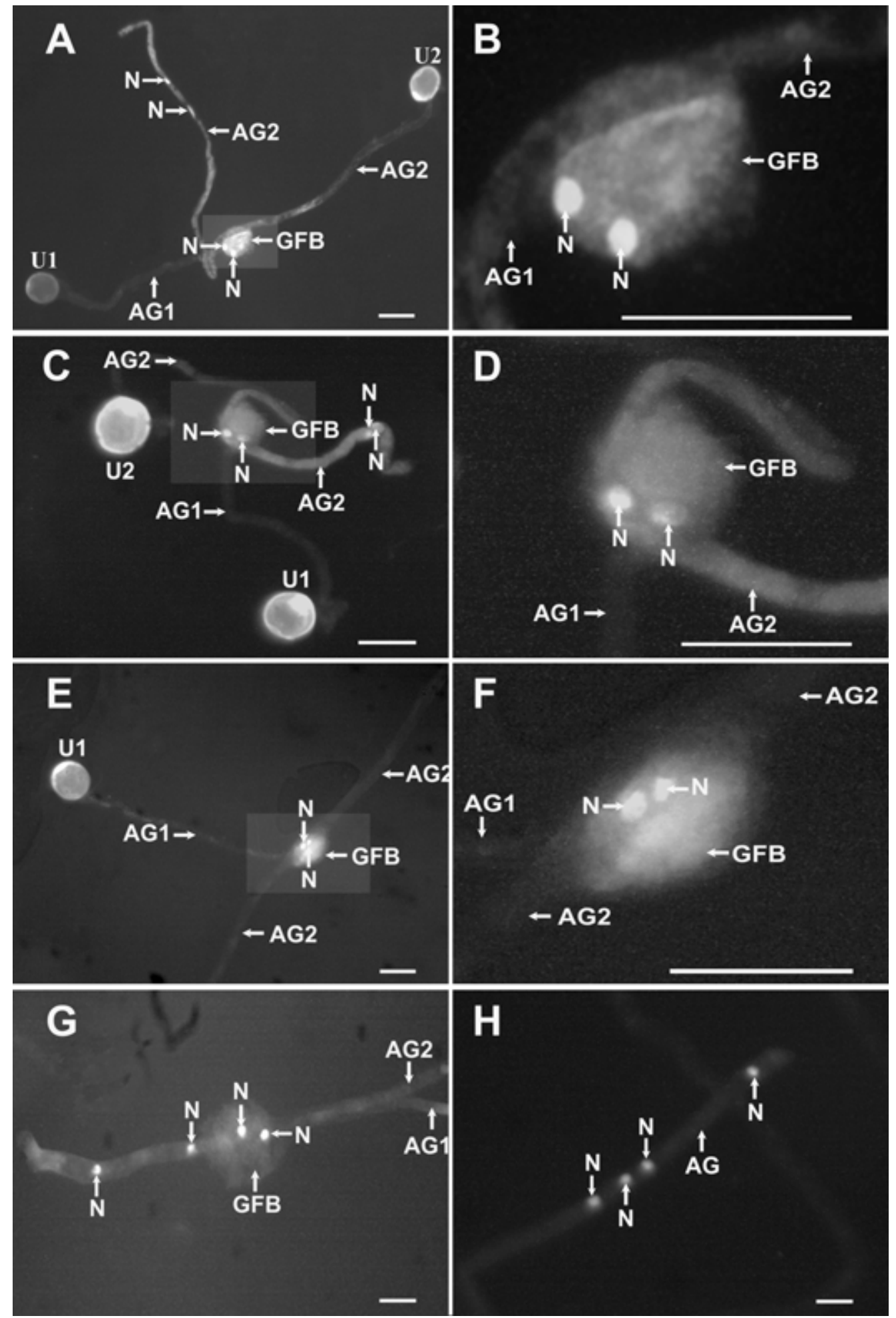

Fig. 6. The migration of nuclei in Puccinia triticina strain MBDS-3-115 "tip to side" germ tube anastomosis. A and B, Two nuclei (N) from one urediniospore (U1) present in the germ tube fusion body (GFB) whereas nuclei from the second urediniospore (U2) were randomly distributed in the germ tube (AG2). C and D, The migration of one nucleus (N, AG1) from the germ tube fusion body (GFB) into the second germ tube (AG2) while two nuclei originating from the second germ tube (AG2) were positioned far away from the GFB. E and F, The migration of both nuclei from AG1 into AG2 through a GFB. G and H, The presence of germ tubes with four nuclei after germ tube anastomosis. U, urediniospore: AG, aerial germ tube. Bar represents $15 \mu \mathrm{m}$. Urediniospores are stained with DAPI. The pictures were taken $24 \mathrm{~h}$ after germination with a Leica360 black and white camera and processed in Photoshop. The boxed regions in A, C, and E are enlarged and shown in $\mathbf{B}, \mathbf{D}$, and $\mathbf{F}$, respectively. 
simply inoculating a mixture of different races on susceptible hosts and collecting urediniospores from resulting pustules. The involvement of the vegetative fusion and subsequent exchange of nuclei between different races of cereal rusts has been hypothesized $(6,22,32)$. Nevertheless, the evidence demonstrating the involvement of germ tube anastomosis in somatic hybridization of
P. triticina is still missing due to the limited number of isolates used in previous studies and the relatively poor cytological details available on the process of germ tube anastomosis in P. triticina. In this study, germ tube anastomoses were observed both in vitro and in vivo and the process was very similar between the in vitro and in vivo conditions. Leaf topology could affect germ tube
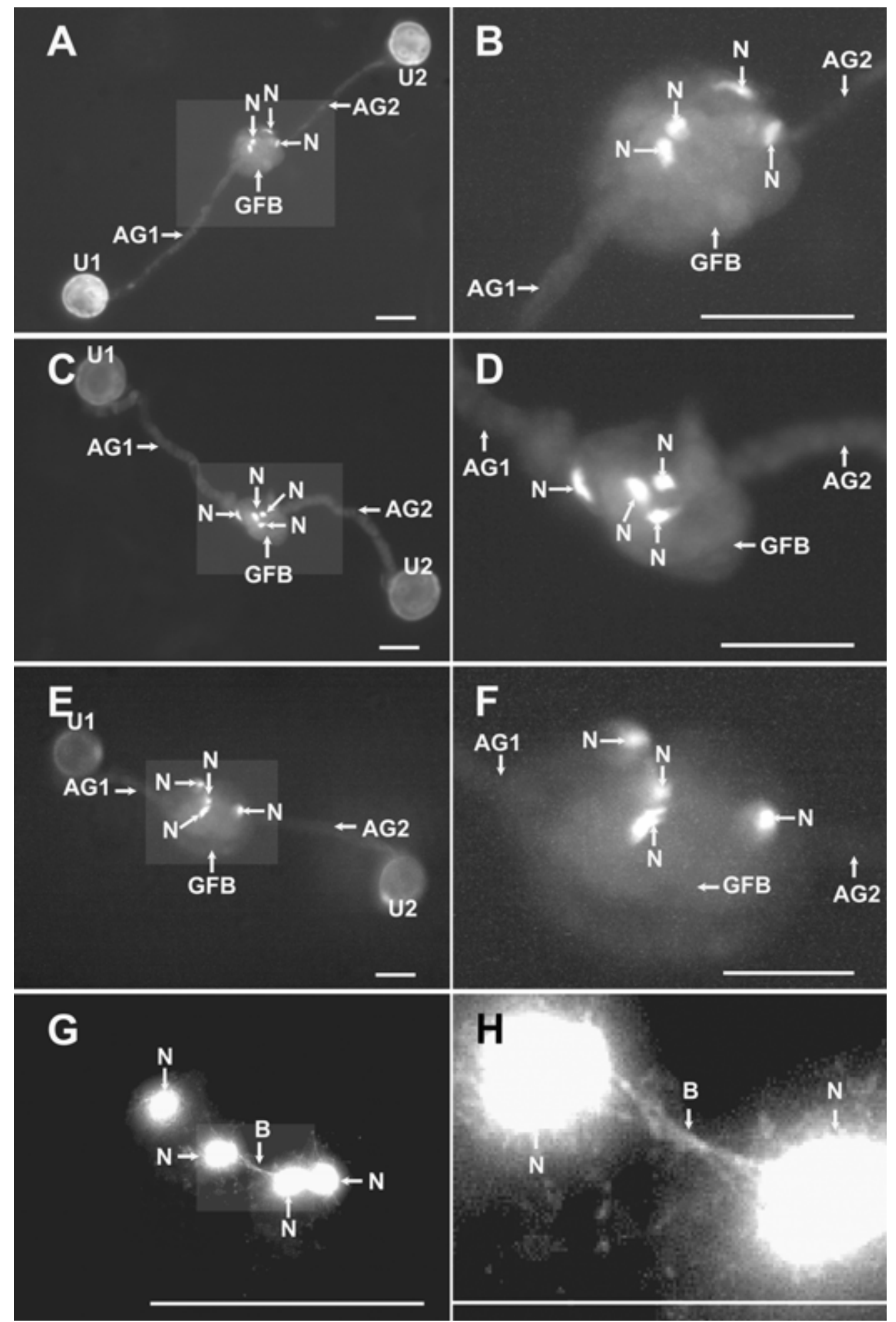

Fig. 7. The migration of nuclei in Puccinia triticina strain MBDS-3-115 "tip to tip" germ tube anastomosis. A and B, The location of nuclei from two urediniospores (U1 and U2) during the early phase of germ tube anastomosis. C and D, The rearrangement of nuclei from two urediniospores (U1 and U2) during germ tube anastomosis. $\mathbf{E}$ and $\mathbf{F}$, The pairing of nuclei from two urediniospores during anastomosis (U1 and U2). $\mathbf{G}$ and $\mathbf{H}$, The occurrence of "bridge-like" structures between nuclei during anastomosis. The boxed regions in $\mathbf{A}, \mathbf{C}, \mathbf{E}$, and $\mathbf{G}$ are enlarged and shown in $\mathbf{B}, \mathbf{D}, \mathbf{F}$, and $\mathbf{H}$. U, urediniospore; AG, aerial germ tube; GFB, germ tube fusion body; N, nuclei; B, the "bridge-like" structure connecting two nuclei. Bar represents $15 \mu$ m. Urediniospores are stained with DAPI. Pictures were taken $24 \mathrm{~h}$ after germination with a Leica360 black and white camera and processed in Photoshop. 
anastomosis, since germ tubes often grow pressed to the leaf cuticle until stomata is encountered and penetrated. Anastomosis could also occur between hyphae inside the leaf tissue after germ tubes have penetrated the leaf surface. These aspects could be investigated in future studies by detailed cytological studies of infections on whole leaves.

The formation of GFBs and germ tube anastomosis were found in 27 P. triticina virulence phenotypes suggesting that this process to be common and biologically significant in $P$. triticina. Furthermore, the migration of nuclei between each pair of germ tubes was observed in both "tip to side" and "tip to tip" germ tube anastomosis. Two dikaryotic nuclei $(\mathrm{N}+\mathrm{N})$ from one germ tube migrated into the second germ tube through the GFB in "tip to side" germ tube anastomosis (Fig. 6A to D) whereas nuclei $(\mathrm{N}+\mathrm{N}$ plus $\mathrm{N}+\mathrm{N}$ ) from both germ tubes involved in anastomosis moved into the fused GFB and were positioned in close proximity in "tip to tip" germ tube anastomosis (Fig. 6E to $\mathrm{H}$ ). The presence of two sets of nuclei $(\mathrm{N}+\mathrm{N}+\mathrm{N}+\mathrm{N})$ in fused germ tubes supported the observation made by Nelson et al. (22) who found that a new race originated from a mixture of two distinct races of $P$. graminis often contained three or four nucleate urediniospores and hyphal cells. This result filled the gap between the studies of Rodenhiser and Hurd-Karrer (28) and Nelson et al. (22) and clearly demonstrated that nuclear migration was involved during germ tube anastomoses in $P$. triticina which led to the formation of multinucleate germ tubes and GFBs. Germ tube anastomosis in $P$. triticina can create an association of nuclei from different germ tubes and result in the production of new heterokaryotic phenotypes.

It was previously not known whether both self- and non-self anastomosis could occur in $P$. triticina (28). In this study, urediniospores of $P$. triticina with different virulence phenotypes were stained with either DAPI or Nuclear Yellow and then mixed and spread out for germination (Fig. 8B and D). Nuclei from germ tubes of different $P$. triticina virulence phenotypes were found to come into close proximity in GFBs and became paired during germ tube anastomosis. The presence of two sets of nuclei of different origins, demonstrated by the differential nuclear staining, in the same GFB or germ tube during germ tube anastomosis indicates that both self and non-self anastomosis can occur during germ tube anastomosis in $P$. triticina. This is the first cytological evidence demonstrating the asexual fusion between genetically different individuals in cereal rusts in which nuclei from isolates of different virulence phenotypes were associated.

The non-self anastomosis in P. triticina is different from that found in the fusion caused by conidial anastomosis tubes in Colletotrichum and Neurospora which only occur among genetically identical individuals $(25,26)$. To date, studies on the non-self anastomosis, defined as vegetative compatibility groups, have been mainly focused on necrotrophic fungi such as Alternaria brassicicola, Fusarium graminearum, and Rhizoctonia solani $(5,20,23)$. It involves the fusion between mature hyphae from nonidentical individuals without the formation of either CATs or GFBs (14). In P. triticina, germ tube anastomosis is related to GFBs produced at tips of germ tubes during the germination of urediniospores and both self and non-self germ tube anastomosis can occur. These characteristics make $P$. triticina system also differ from the non-self anastomosis found in A. brassicicola since self-anastomosis does not occur between conidia or young germlings of $A$. brassicicola (5).

Nelson et al. (22) reported that new heterokaryons isolated from the mixture of two $P$. graminis races were more virulent than the original races comprising the mixture and they produced urediniospores with three or four nuclei. This result suggests that multinucleate GFBs or germ tubes formed during the germ tube anastomosis are most likely viable and pathogenic. The non-self anastomosis and the grouping of nuclei from $P$. triticina isolates of different virulence phenotypes in fused GFBs could provide a new mean, aside from the sexual process, of bringing together different virulence genes and creating a heterokaryon with a

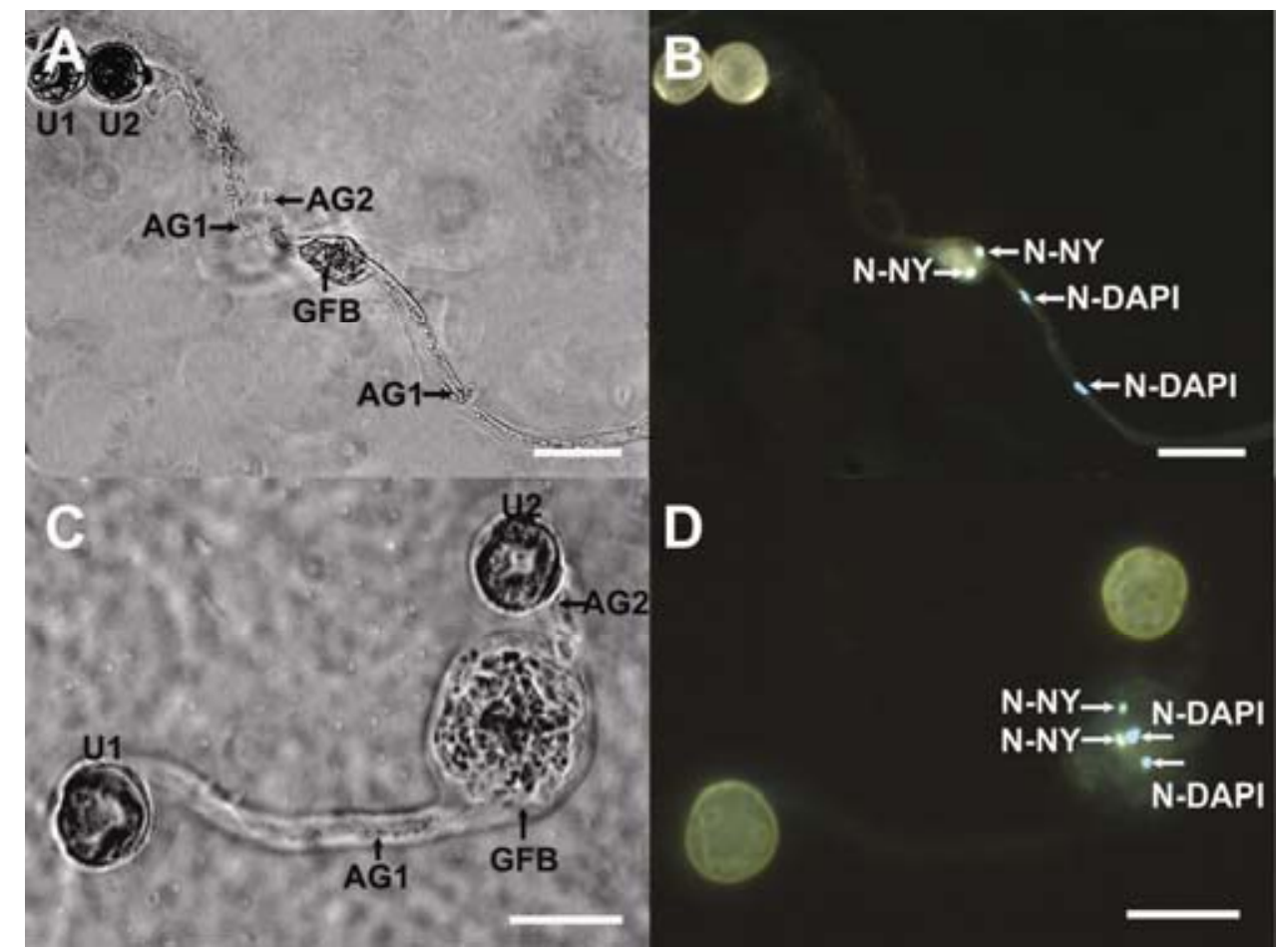

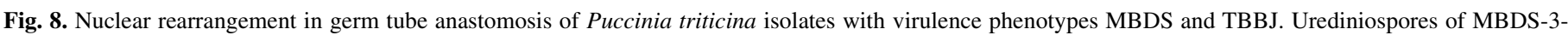

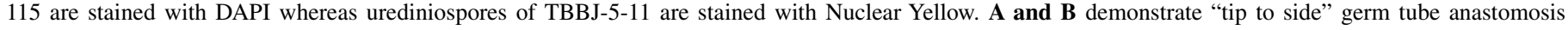

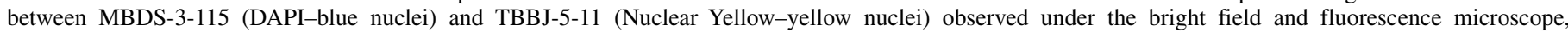

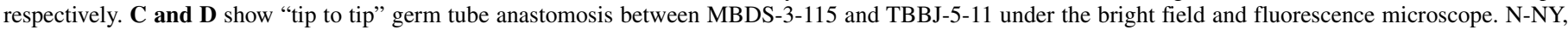

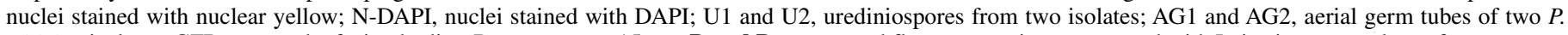
triticina isolates; GFB, germ tube fusion bodies. Bar represents $15 \mu \mathrm{m}$. B and D are merged fluorescence images created with Leica image overlay software. 
broader virulence spectrum than the original isolates. Furthermore, Nelson et al. (22) found that the newly produced heterokaryons differed in the number of nuclei within cells and the percentage of urediniospores with three to four nuclei was higher in early generations than in subsequent generations in which the normal dikaryotic nuclear configuration was restored. This suggests that the multinucleate condition formed during germ tube anastomosis was unstable and the complete reversion to dikaryophase may occur at various points in the life cycle of $P$. triticina.

A logical question to ask is whether subsequent nuclear exchange is involved after the association of different nuclei within fused GFBs. In our study, connections between nuclei resembling bridge-like structure were observed in two GFBs containing four DAPI-stained nuclei. The function of these bridge-like structures in germ tube anastomosis of $P$. triticina is currently unknown. It is not clear whether such structures are involved in the self or nonself fusion since we were unable to track the origin of parent germ tubes when bridge-like structures were observed. Nevertheless, these bridge-like structures were very similar to the chromosomal bridges commonly seen in human cancer cells exhibiting gross chromosomal alterations such as translocations, deletions, or gene amplifications $(17,30)$.

In summary, our study demonstrated that the formation of GFBs was common in P. triticina and germ tube anastomosis was dependent upon the involvement of at least one GFB. Formation of GFBs was dependent on the spore density and length of illumination period. GFBs attached to either the side of another germ tube or directly to another GFB. The germ tube appeared to coil inside the GFB in response to fusion with a second germ tube. Nuclei migrated into close proximity inside GFBs once fusion had occurred. The exchange of cytoplasm and nuclei between different germ tubes occurred during germ tube anastomosis. We reported the first investigation of both self and non-self germ tube anastomosis in $P$. triticina and provided the evidence for a direct correlation between germ tube anastomosis and the rearrangement of nuclei which suggested that germ tube anastomosis could directly contribute to the parasexual recombination in $P$. triticina. The occurrence of nuclear rearrangement in $P$. triticina and the close association between nuclei from different $P$. triticina virulence phenotypes indicates that heterokaryosis and new virulence combinations could be generated through germ tube anastomosis. Our study raises the question of whether chromosomal exchange between nuclei is involved or does the reassortment of whole nuclei and subsequent nuclear reduction lead to the somatic recombination. Such questions could hopefully be addressed through living cell imaging and fluorescence in situ hybridization in future studies.

\section{ACKNOWLEDGMENTS}

This work was supported by funding from Agriculture and Agri-Food Canada genomics project. X. Wang is the recipient of an NSERC visiting fellowship in AAFC laboratories.

\section{LITERATURE CITED}

1. Barr, R., Caldwell, R. M., Amacher, R. H. 1964. An examination of vegetative recombination of urediniospore color and virulence in mixtures of certain races of Puccinia recondita. Phytopathology 54:104-109.

2. Bridgemon, G. H., and Wilcoxson, R. D. 1959. New races from mixtures of urediniospores of varieties of Puccinia graminis. Phytopathology 49:428-429.

3. Burdon, J. J., Marshall, D. R., and Luig, N. H. 1981. Isozyme analysis indicates that a virulent cereal rust pathogen is a somatic hybrid. Nature 293:565-566

4. Burdon, J. J., and Silk, J. 1997. Sources and patterns of diversity in plantpathogenic fungi. Phytopathology 87:664-669.
5. Craven, K. D., Velez, H., Cho, Y. G., Lawrence, C. B., and Mitchell, T. K. 2008. Anastomosis is required for virulence of the fungal necrotroph Alternaria brassicicola. Eukary. Cell 7:675-683.

6. Ellingboe, A. H. 1961. Somatic recombination in Puccinia graminis var. tritici. Phytopathology 51:13-15.

7. French, R. C., and Gallimore, M. D. 1972. Stimulation of germination of urediniospores of stem rust of wheat in the pustule by n-nonanal and related compounds. J. Agric. Food Chem. 20:421-423.

8. Glass, N. L., Rasmussen, C., Roca, M. G., and Read, N. D. 2004. Hyphal homing, fusion and mycelial interconnectedness. Trends Microbiol. $12: 135-141$

9. Goddard, M. V. 1976. The production of a new race, 105E 137 of Puccinia striiformis in glasshouse experiments. Transactions of the Brit. Mycol. Soc. 67:395-398.

10. Knott, D. R. 1989. The Wheat Rusts-Breeding for Resistance. SpringerVerlag, Berlin.

11. Kolmer, J. A. 1996. Genetics of resistance to wheat leaf rust. Annu. Rev. Phytopathol. 34:435-455.

12. Kolmer, J. A. 2005. Tracking wheat rust on a continental scale. Curr. Opin. Plant Biol. 8:441-449.

13. Kolmer, J. A., Liu, J. Q., and Sies, M. 1995. Virulence and molecular polymorphism in Puccinia recondita f. sp. tritici in Canada. Phytopathology 85:276-285.

14. Leslie, J. F. 1993. Fungal vegetative compatibility. Annu. Rev. Phytopathol. 31:127-150.

15. Little, R., and Manners, J. G. 1969. Somatic recombination in yellow rust of wheat (Puccinia striiformis). I. The production and possible origin of two new physiological races. Trans. Br. Mycol. Soc. 53:251-258.

16. Manners, J. G., and Bampton, S. S. 1957. Fusion of urediniospore germ tubes in Puccinia graminis. Nature 179:483-484.

17. Masuda, A., and Takahashi, T. 2002. Chromosome instability in human lung cancers: Possible underlying mechanisms and potential consequences in the pathogenesis. Oncogene 21:6884-6897.

18. McCallum, B. D., and Seto-Goh, P. 2004. Physiological specialization of wheat leaf rust (Puccinia triticina) in Canada in 2001. Can. J. Plant Pathol. 26:109-129.

19. McCallum, B. D., and Seto-Goh, P. 2005. Physiological specialization of wheat leaf rust (Puccinia triticina) in Canada in 2002. Can. J. Plant Pathol. 27:90-99.

20. McCallum, B. D., Tekauz, A., and Gilbert, J. 2001. Vegetative compatibility among Fusarium graminearum (Gibberella zeae) isolates from barley spikes in southern Manitoba. Can. J. Plant Pathol. 23:83-87.

21. Nelson, R. R. 1956. Transmission of factors for urediniospore color in Puccinia graminis var. tritici by means of nuclear exchange between vegetative hyphae. Phytopathology 46:538-540.

22. Nelson, R. R., Wilcoxson, R. D., and Christensen, J. J. 1955. Heterokaryosis as a basis for variation in Puccinia graminis var. tritici. Phytopathology 45:639-643.

23. Ogoshi, A. 1987. Ecology and pathogenicity of anastomosis and intraspecific groups of Rhizoctonia solani Kühn. Annu. Rev. Phytopathol. 25:125-143.

24. Park, R. F., Burdon, J. J., and Jahoor, A. 1999. Evidence for somatic hybridization in nature in Puccinia recondita f. sp. tritici, the leaf rust pathogen of wheat. Mycol. Res. 103:715-723.

25. Roca, M. G., Davide, L. C., Mendes-Costa, M. C., and Wheals, A. 2003. Conidial anastomosis tubes in Colletotrichum. Fungal Genet. Biol. 40:138-145.

26. Roca, M. G., Arlt, J., Jeffree, C. E., and Read, N. D. 2005. Cell biology of conidial anastomosis tubes in Neurospora crassa. Eukary. Cell 4:911-919.

27. Roca, M. G., Read, N. D., and Wheals, A. 2005. Conidial anastomosis tubes in filamentous fungi. FEMS Microbiol. Lett. 249:191-198.

28. Rodenhiser, H. A., and Hurd-Karrer, A. M. 1947. Evidence of fusion bodies from urediniospore germ tube of cereal rusts on nutrient solution agar. Phytopathology 46:744-756.

29. Samborski, D. J. 1985. Wheat leaf rust. Pages 39-59 in: The Cereal Rusts. Vol. 2. A. P. Poelfs and W. R. Bushnell, eds. Academic Press, Orlando, FL.

30. Shimizu, N., Shingaki, K., Kaneko-Sasaguri, Y., Hashizume, T., and Kanada, T. 2004. When, where and how the bridge breaks: Anaphase breakage plays a crucial role in gene amplification and HSR generation. Exp. Cell Res. 302:233-243.

31. Watson, I. A. 1981. Wheat and its rust parasites in Australia. Pages 129147 in: Wheat Science-Today and Tomorrow. L. T. Evans and W. J. Peacock, eds. Cambridge University Press, Cambridge.

32. Wilcoxon, R. D., Tuite, J. F., and Tucker, S. 1958. Urediniospores germ tube fusions in Puccinia graminis. Phytopathology 48:358-361.

33. Wright, R. G., and Lennard, J. H. 1980. Origin of new race of Puccinia striiformis. Trans. Br. Mycol. Soc. 74:283-287. 